sequences on extinction performance was shown by the significant groups component $(F=19.04$, df $=4 / 50, p<.01)$ and the $G$ by $T$ interaction $(F=6.00$, df $=12 / 150$, $\mathrm{p}<.01)$. $T$ tests between individual pairs of groups based on each S's average speed throughout extinction were also performed. It was found that Group SM was more resistant to extinction than any other group ( $<<.001)$; Group ML was more resistant than $M M(p<.01)$ and $L M(p<.001)$ but was borderline with respect to MS $(.05<\mathrm{p}<.10)$. None of the other differences tested was significant.

\section{DISCUSSION}

In general, the ordering of the groups in extinction was in close agreement with predictions from sequential theory. For the three groups which received equal rewards on Trial 2 of each training day (SM, MM, LM), small reward on Trial 1 tended to increase $R n$ as predicted, although the difference between MM and LM was not significant by the $t$ test. This finding agrees with the sequential interpretation that different aftereffects are generated by the Trial 1 reward amounts, and conditioned on Trial 2 by (medium) reward, and produce resistance to extinction inversely proportional to the Trial 1 amount.

When the same aftereffect (SRM) was generated on Trial 1 in Groups MS, MM, and ML, but conditioned by varying amounts of reward on Trial 2, the prediction that greater $\mathrm{Rn}$ should obtain with larger Trial 2 reward was only partially verified. Group ML was most resistant, as predicted, but Groups MM and MS did not differ. ${ }^{3}$ The effect of the magnitude variable appeared to be much less when reward was varied on Trial 2 rather than on Trial 1.

The large differences in extinction performance between groups which received the same total amount of reward each day but differed in the reward sequence (ML vs LM; SM vs MS) were expected by the hypothesis and verify the previous results of Leonard (1969) for groups trained SL and LS. The former groups in each comparison have the double advantage in that a smaller Trial 1 aftereffect is conditioned more strongly by a larger Trial 2 reward.

\section{REFERENCES}

CAPALDI, E. J. A sequential hypothesis of instrumental learning. In $\mathrm{K}$. W. Spence and J.T. Spence (Eds.), The psychology of learning and motivation: Advances in research and theory.

Vol. 1. New York: Academic Press, 1967. Pp. 67-156.

CAPALDI, E. J., \& SPIVEY, J. E. Stimulus consequences of reinforcement and nonrein forcement: Stimulus traces or memory. Psy chonomic Science, 1964, 1,403-404.

LEONARD, D. W. Amount and sequence of reward in partial and continuous reinforcement. Journal of Comparative \& Physiological Psychology, 1969, 67, 204-211.
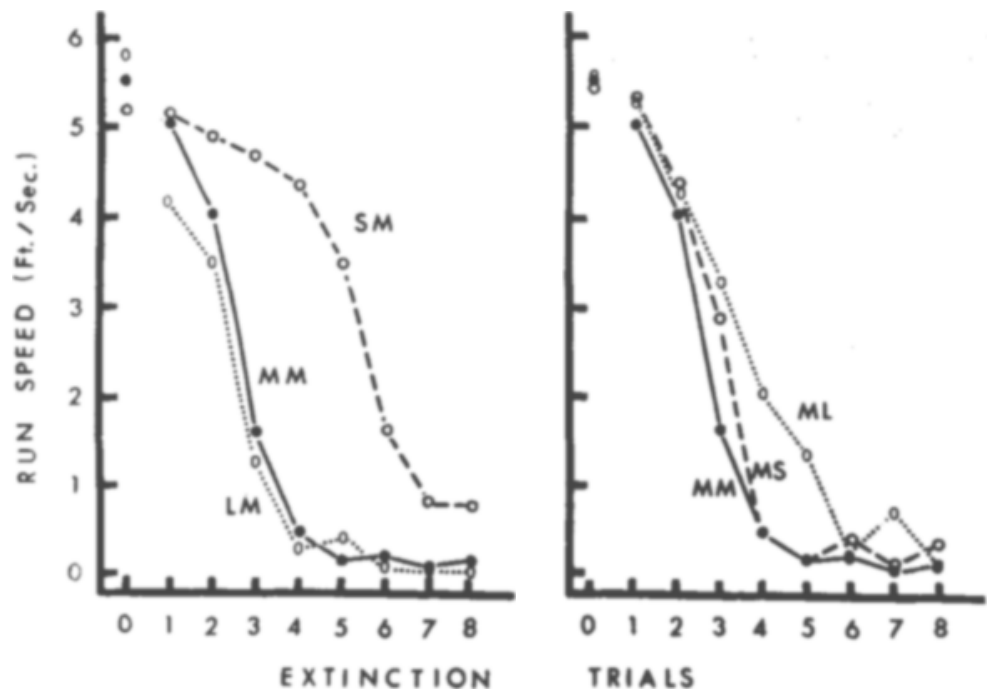

Fig. 2. Extinction performance of the five experimental groups on the nine extinction trials. Group MM is plotted twice for comparison purposes.

SHEFFIELD, V. F. Extinction as a function of partial reinforcement and distribution of practice. Joumal of Experimental Psy chology, $1949,39,511-526$.

\section{NOTES}

1. This investigation was supported in part by Research Grants MH-15704-01 and MH-10825-03 from the National Institute of Mental Health, and by the Center for Naval Analysis of the University of Rochester, Contract N00014-68A-0091. Such support does not imply endorsement of the content by the Navy.

2. The term "aftereffect" is used only for convenience, and is not meant to imply equivalence to the short-term trace relating to food in the teeth, etc., as originally conceived by Sheffield (1949). Capaldi feels the stimulus involved is long-term, perhaps more akin to a memory than to a simple trace (e.g., Capaldi \& Spivey, 1966), but its precise status is still in doubt.

3. A third replication using standard Noyes pellets and involving only the three groups in question resulted in the predicted extinction order, $\mathrm{ML}>\mathrm{MM}>\mathrm{MS}$; however, the difference between MM and MS again did not reach accepted levels of significance. Group ML was significantly more resistant than either of the other two groups as predicted. These Ss were run by Linda Leighton and Betsey Bensen.

\title{
Performance under massed or spaced extinction following different sequences of varied reward training
}

\section{DALE W. LEONARD, RICHARD ALBIN, and MARK LEBOWITZ, University of Rochester, Rochester, N.Y. 14627}

Rats were run in a straight alley to test an unusual prediction by sequential theory to the effect that a varied reward group trained with an SL reward sequence would tend to be more resistant than an $L S$ group under massed extinction, but less resistant under spaced extinction. A significant interaction between sequence and type of extinction was obtained. Also, a random varied reward group exhibited the greatest resistance under either extinction condition.

Leonard (1969) has reported that a varied-reward group given two daily training trials each day in a small-large reward sequence (SL) was more resistant to extinction than a group trained LS each day. This result was interpreted within the framework of Capaldi's (1967) sequential learning theory. 
When a two-trial reward sequence such as SL or LS is repeated over a series of days, however, the only real difference between the two sequences is the time period which elapses between the transition from small to large reward (T1) and the transition from large to small reward (T2). In Group SL, T1 is a matter of minutes whereas $\mathrm{T} 2$ is $24 \mathrm{~h}$, the time between daily sessions. In Group LS, T2 is very short whereas T1 is $24 \mathrm{~h}$.

According to sequential theory, part of the stimulus component of an aftereffect is the age of that aftereffect (Capaldi, Leonard, \& Ksir, 1968). Thus, if an S has been repeatedly reinforced in the presence of a small-reward aftereffect (SRS) which is only $2 \mathrm{~min}$ old, the greatest amount of generalization will be to a 2-min-old extinction aftereffect(SN). Therefore, since an LS group does receive an SL transition across days, its subsequent poor extinction performance cannot be attributed to the absence of SL transitions, but rather to the fact that it receives its SL transition at a time interval inappropriate to the time interval employed in extinction. That is, the previous superiority of SL over LS sequences was demonstrated by Leonard using massed extinction trials. The 2-min SRS aftereffect conditioned in Group SL provides more generalized habit to a $2-\mathrm{min}$ SN than does the 24-h SRS aftereffect conditioned in Group LS.

Suppose, however, that extinction were carried out at one trial per day. Here, the hypothesis predicts that Group LS would prove to be more resistant than Group SL. This would show up as a significant Reward Sequence by Extinction Condition interaction in an analysis of variance. The present experiment was designed to test this somewhat unusual prediction by the sequential hypothesis. A third group which received both SL and LS sequences each day in a semirandom order was also run in order to check on possible side effects of the highly ordered SL and LS sequences. This group, designated Group $R$, is more nearly equivalent to the varied magnitude groups employed by other investigators. All three groups were then extinguished using either massed or widely spaced trials.

\section{SUBJECTS}

The Ss were 36 male Holtzman rats, approximately 90 days old on arrival.

\section{APPARATUS}

A $36 \times 3 \% 2 \times 4$ in. straight alley runway was used. The alley was constructed of plywood, covered with a hinged top of clear Plexiglas, and was painted flat black. A wooden baffle at the end of the alley concealed an aluminum goal cup into which Noyes $.045-\mathrm{g}$ pellets could be placed. A guillotine door $12 \mathrm{in}$. from the end of the alley was used to prevent retracing from the goal area. The sliding door on the 12-in. start section activated a Hunter Klockcounter which recorded starting latencies in the following 12-in. portion of the alley to the nearest $.01 \mathrm{sec}$. A photocell system connected to a second Klockcounter recorded run times in the final 12 -in. portion of the alley.

\section{PROCEDURE}

After 1 week of ad lib feeding, Ss were placed on a 14-g-per-day deprivation schedule. Water was always available in the home cages. During the initial 10 days of deprivation Ss were handled individually and trained to eat the Noyes pellets by placing them in the baited goal area with the guillotine door closed.

Following this pretraining period, Ss were randomly divided into three groups of $12 \mathrm{Ss}$ each and trained to traverse the alley at the rate of two trials per day for a 10-day period. Group SL received a small reward (2 pellets) on its first daily trial followed by large reward (16 pellets) on the second daily trial. Group LS received the opposite sequence. Group $R$ received an SL sequence on Days 1 , $3,4,6$, and 10 , and an LS sequence on the other 5 days. Ss were run in squads during training, each squad consisting of one $S$ from each of the three groups, so that the intertrial interval (ITI) was determined by the time needed to run the other two Ss in the squad (approximately $2 \mathrm{~min}$ ). Training was staggered slightly so that only two squads began the extinction phase on a given day.

After training, half the $\mathrm{Ss}$ in each group were extinguished with a massed trial procedure and the other half with spaced trials. In the massed extinction condition $S$ s were run in squad rotation as in training. Under spaced conditions, Ss received one extinction trial per day. All Ss ran nine extinction trials and were confined to the empty goal area for a period of $30 \mathrm{sec}$ on each occasion. One $S$ in the SL spaced extinction condition became ill suddenly and was dropped from the extinction phase.

\section{RESULTS}

The start and run times obtained during both the acquisition and extinction phases were converted to speed scores (in./sec) for purposes of statistical analysis. Statistical results for both sections are presented but only run speeds are shown.

The last 2 days of acquisition training, Days 9 and 10, were used to determine whether or not the three acquisition conditions produced different speeds, and whether or not Ss patterned their speeds on the two daily trials in accord with the reward sequences employed. An analy sis of variance with groups (G), days (D), and trials within a day (T) was used for this purpose. None of the Fs approached the .05 significance level in either the start or run speed analyses.
Plots of group speeds on these trials bore out the statistical result that the groups did not differ just prior to extinction.

Somewhat slower speeds by Group $\mathbf{R}$ during the early phases of training, however, prompted us to do an analysis of the entire 10-day training phase. Logan (1960) has hypothesized that competing anticipatory goal responses (rg's) may produce slower running in varied magnitude groups. Slower running by Group $R$, with its unpredictable reward pattern, would support such an hypothesis. However, a one-way analysis using each S's average speed throughout training produced no differences in either the start $(F=2.39, \mathrm{df}=2 / 33, \mathrm{p}>.10)$ or the run $(F=1.11, \mathrm{df}=2 / 33, \mathrm{p}>.25)$ sections.

In the analysis of extinction performance, the hypothesis to be evaluated was that SL would tend to outperform LS under massed extinction conditions whereas LS would predominate under spaced extinction. The analysis was therefore performed on the last eight extinction trials of Groups SL and LS, excluding Group R, with the expectation that a significant Groups by Massed or Spaced Extinction Condition (G by E) interaction would result. Figure 1 shows that the running speeds of the SL and LS groups were ordered in agreement with the hypothesis. Start speeds were similar except that the superiority of Group LS over Group SL under spaced extinction was less clear-cut.

The $\mathrm{G}$ by $\mathrm{E}$ interaction was significant in both the $\operatorname{start}(F=4.69, \mathrm{df}=1 / 19, \mathrm{p}<.05)$ and $\operatorname{mun}(F=9.18, d f=1 / 19, p<.01)$ sections as predicted. The only other variance components to reach significance were those reflecting the slower extinction under spaced conditions, and for the decrement in speed across extinction trials (both ps <.01)

The Mann-Whitney test was then applied to the run speeds of individual pairs of groups within the massed or spaced extinction conditions. For massed extinction groups, Group R outperformed both SL $(p<.05)$ and $L S(p<.01)$ and $S L$ outperformed LS $(p<.05)$. For spaced extinction groups. $R$ outperformed SL $(p<.05)$ but was borderline with respect to LS $(.05<p<.10)$, as was the SL-LS difference $(.05<\mathrm{p}<.10)$.

\section{DISCUSSION}

The extinction results clearly supported the sequential theory's prediction that the order of the SL and LS reward groups would reverse as a function of the time interval between extinction trials.

In considering the spaced extinction results, a "common sense" explanation might involve the fact that the LS group always received large reward on the first trial 
of each day during training, and that this may somehow have carried over to the one-trial-per-day extinction. It should be pointed out, however, that this notion is not at all contradictory to sequential theory; in fact, the theory simply translates this general notion into more precise terminology. For example, "first trial of the day" is translated to mean a stimulus aftereffect whose components depend upon the reward event on the previous day, and $S 24 h$, the aftereffect of the passage of time since that event. The fact that large reward then occurs on the first trial by definition conditions this component stimulus to the instrumental response.

In the massed extinction situation, application of the "common sense" explanation becomes less obvious since $S \delta$ were extinguished in one day, rather than in two trial blocks over several days. This, of course, is demanded by the theory in order to avoid periodic contamination produced by spontaneous recovery. The sequential theory explanation, however, is still applicable based on the assumption that $S s$ in Group SL had conditioned a 2-min SRS aftereffect.

Turning now to the random group, the superior extinction performance of Group $R$ under both massed and spaced conditions was somewhat unexpected. Sequential theory predicts such an occurrence only when training is near asymptote such that the summation of habit strength at both SRL and SRS as in Group R would exceed that of a group in which only one of these stimuli was conditioned. Due to the

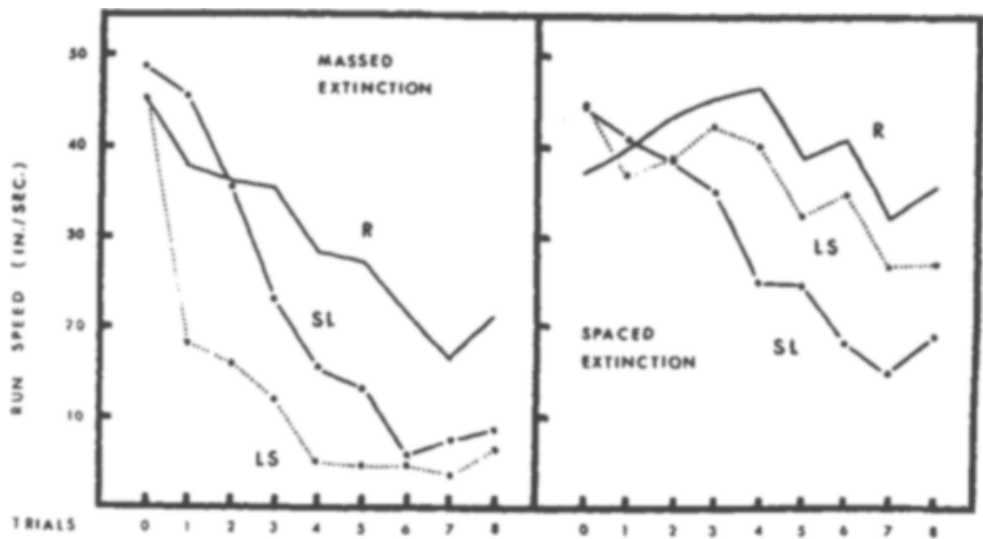

Fig. 1. The effect of massed or spaced extinction on the performance of groups receiving an SL, LS, or random reward sequence.

limited amount of training in the present experiment, however, it was expected that Group R would be intermediate to SL and $\mathrm{LS}$, since it receives an intermediate number of SL transitions. Since this was not the case, it is possible that the irregularity of Group R's schedule enhanced its extinction performance above and beyond that expected from a simple examination of the sequences it received. More work in this direction is indicated.

\section{REFERENCES}

CAPALDI, E. J. A sequential hypothesis of instrumental learning. In $\mathrm{K}$. W. Spence and J. T. Spence (Eds.), The psychology of learning and motivation: Advances in research and theory. Vol. 1. New York: Academic Press, 1967. Pp. 67-156.
CAPALDI, E. J., LEONARD, D. W \& \& KSIR, C. A reexamination of extinction rate in successive acquisitions and extinctions. Journal of Com. parative \& Physiological Psychology, 1968, 66, 128-132.

LEONARD, D. W. Amount and sequence of reward in partial and continuous reinforcement. Journal of Comparative \& Physiological Psy chology, 1969, 67, 204-211.

LOGAN, F. A. Incentive. New Haven: Yale University Press, 1960.

\section{NOTE}

1. This investigation was supported in part by Research Grants MH-15704-01 and MH-10825-03 from the National Institute of Mental Health, and by the Center for Naval Analysis of the University of Rochester, Contract N00014-68A-0091. Such support does not imply endorsement of the content by the Navy.
Donald $W$. Taylor became Dean of the Graduate School, Yale University on July 1.Formerly he was Director of the Institute of Social Science and Chairman of the Department of Psychology, Yale University.

Katherine E. Baker has left State University College at Buffalo to become Professor of Psychology, Hood College, Frederick, Maryland.

S. Joyce Brotsky returns to San Fernando Valley State College as Associate Professor of Psychology after a recent leave of absence, supported by an NIH Career Development Research Fellowship in Harvard

\section{University's Department of Social Relations.}

Donn Byrne, formerly Professor of Psychology at The University of Texas, Austin, will become Professor of Psychology at Purdue University in September 1969.

Peter D. McCormack, Professor of Psychology, Carleton University, Ottawa, Canada, will spend the 1969-70 academic year on sabbatical at the Human Performance Center of the University of Michigan.

Robert H. Pollack, formerly Deputy Director of Research, Institute for Juvenile Research, joined the faculty of the University of Georgia in April, 1969, as Professor of Psychology.
An undergraduate major, leading to the BA or BS in Animal Behavior has been announced by Bucknell University. The program is an interdisciplinary curriculum offered by the departments of biology and psychology. The basic curriculum includes work in organic chemistry, biochemistry, embryology, animal physiology, ecology and evolution, animal behavior, physiological psychology, and learning and conditioning. Because the University operates on a "4-1-4" semester plan, students are free during January for field or additional laboratory work. The Chairman of the interdisciplinary committee administering the program is Professor Douglas K. Candland. 